\title{
Theoretical and numerical characterization of the thermal conductivity of carbon ceramic material from realistic microscale imaging
}

\author{
Pavel V. Prosuntsov ${ }^{1, *}$, and Nikita Y. Taraskin ${ }^{1}$ \\ ${ }^{1}$ Bauman Moscow State Technical University, 105005 Moscow, Russia
}

\begin{abstract}
The paper is concerned with the theoretical and numerical characterization of the thermal conductivity of porous carbon ceramic materials. The material structure models are created based on 3D computer tomography (CT) images. The temperature functions of the molecular and effective thermal conductivity for the porous carbon-ceramic material were obtained. It was investigated, whether a simplified model could be used to estimate the thermal conductivity. The thermal conductivity data obtained with the computer tomography method and with the proposed simplified model are compared.
\end{abstract}

\section{Subject of research}

Carbon-ceramic composite materials (CCCM) hold a prominent position in the space rocket engineering. They are used to make the spacecraft frames temperature-, impact- erosionand chemically resistant, protecting them from the high-speed gas flow [1]. State-of-the-art CCCM [1] retain their efficiency at temperatures up to $2000 \mathrm{~K}$ and possess sufficiently high mechanical properties. Despite this CCCM cannot be employed as the mass efficient thermal protection due to their high density (up to $2000 \mathrm{~kg} / \mathrm{m}^{3}$ ) and high thermal conductivity (up to $60 \mathrm{~W} /(\mathrm{m} \cdot \mathrm{K})$ ) [2]. Thus, porous CCCM with a relatively low density and a far smaller thermal conductivity appear to have a great potential $[3,4]$.

However, the data on the porous CCCM properties, especially with regard to their thermal conductivity coefficient - the key operation parameter, are practically non-existent. Experiments to estimate the thermal conductivity coefficient of such high-temperature CCCM are highly challenging. Moreover, the experiments can determine only the so-called effective thermal conductivity coefficient, which estimates the total heat transfer including both conductive heat transfer along the reinforcement and radiative heat transfer in the pores. The conductive heat transfer is described by means of the molecular thermal conductivity coefficient [5]. Therefore, at this stage of research it appears reasonable to perform the theoretical and numerical estimation of the porous CCCM thermal conductivity separating the heat transfer constituents.

\footnotetext{
* Corresponding author: pavel.prosuntsov@mail.ru
} 
The probable characteristics of porous CCCM with the woven and non-woven reinforcement are theoretically validated in [4]. The heat conductivity analysis of the porous CCCM (with $1000-2000 \mathrm{~kg} / \mathrm{m}^{3}$ density) accounting for the radiative heat transfer in the pores is conducted in [5].

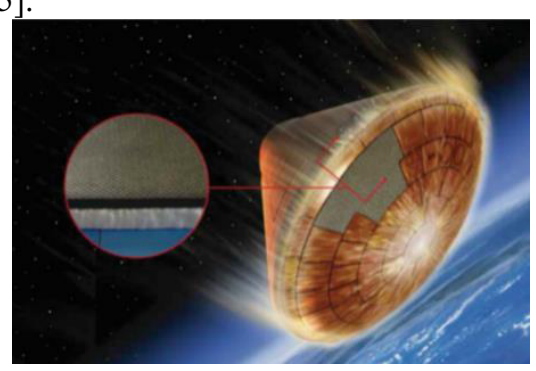

Fig 1. Illustration of a multifunctional HOST heatshield concept for an Earth entry application and a close-up of the carbon-carbon outer layer [2].

The porous CCCM thermal conductivity was estimated based on the numerical simulation of the heat transfer process in the representative volume element. This method employed relatively simple structure models, in the form of intercrossing cylindrical fibres. It was assumed that all the fibres had equal diameter. The study in [5] demonstrated the evident relationship of the effective and molecular thermal conductivity from the temperature and porosity.

\section{Porous CCCM structure investigation}

The reference data regarding the porous CCCM structure were obtained based on the sample microstructure analysis using the Brucker Skyscan 1172 micro-CT scanner. This device uses a multitude of X-Ray images at various angles to create a $3 \mathrm{D}$ model of the sample. Figs. 2-4 present 3D structure models of the porous CCCM with 27-29\% porosity.

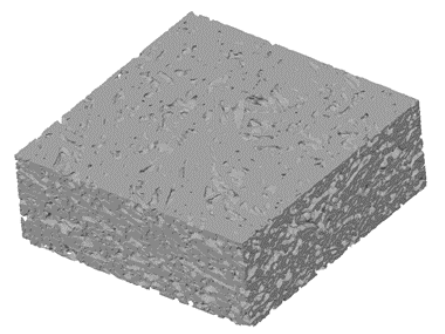

Fig 2. 3D structure model of a porous CCCM sample, obtained with X-ray micro-CT scanning.

Both the original sample model (Fig. 2) and the separate areas (Figs. 3-4) are presented. It is evident that the material structure is significantly different from the models used in [5].

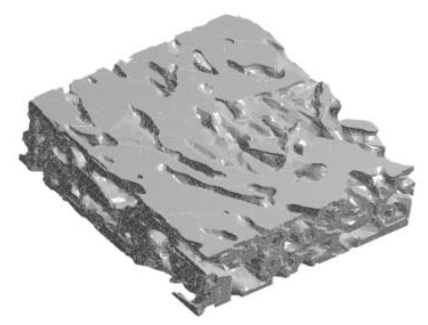

Fig 3. 3D structure model of a porous CCCM sample area, obtained with X-ray micro-CT scanning. 


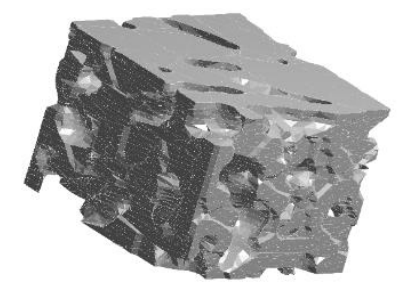

Fig. 4. 3D structure model of a porous CCCM sample area, obtained with X-ray micro-CT scanning.

\section{Determining the thermal conductivity of the porous CCCM by means of X-ray micro-CT scanning method}

In order to determine the effective thermal conductivity coefficient of the porous CCCM an approach similar to [5] was used. First, a finite-element model of a representative volume element was created in the Siemens NX software package (Fig. 5). This model comprised a porous material zone and two contacting plates. The outer surfaces were assumed to have different temperatures. The side surfaces of the plates were assumed to be thermally insulated. The thermal contact between the plates and the porous material fibers was assumed ideal. Additional mirror reflecting surfaces (zero thickness; reflectance $=1$ ) were located on the side surfaces of the porous material. The surfaces of the fibers and plates were assumed to be grey, diffusely reflecting, with the emissivity factor equalling 0.75 . The thermal conductivity of the monolythic CCCM, which was used for calculation, is presented in Fig.6.

The heat transfer simulation in this representative volume element (Fig. 7) resulted in estimating the value of the total heat flux through the porous material and calculating its effective heat transfer coefficient. The resulting temperature function of the molecular and effective heat transfer coefficient of the porous CCCM is presented in Fig.8 (graphs 1 and 2) and corresponds qualitatively to the data in [4].

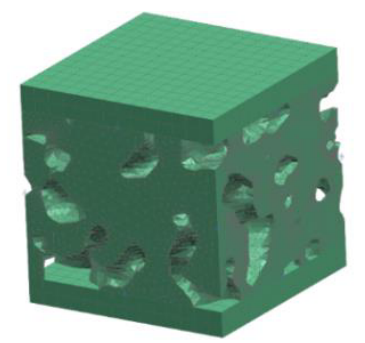

Fig. 5. Finite-element model of the representative volume element of the porous CCCM.

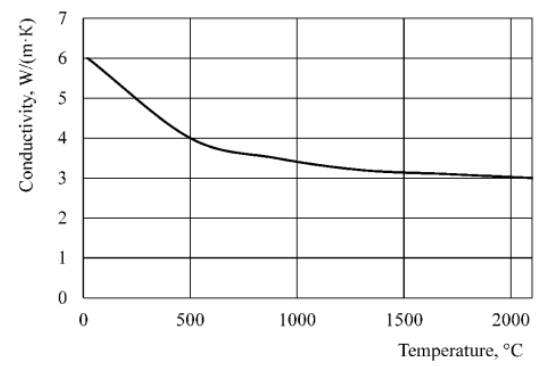

Fig. 6. Thermal conductivity of the monolithic CCCM as a function of temperature. 


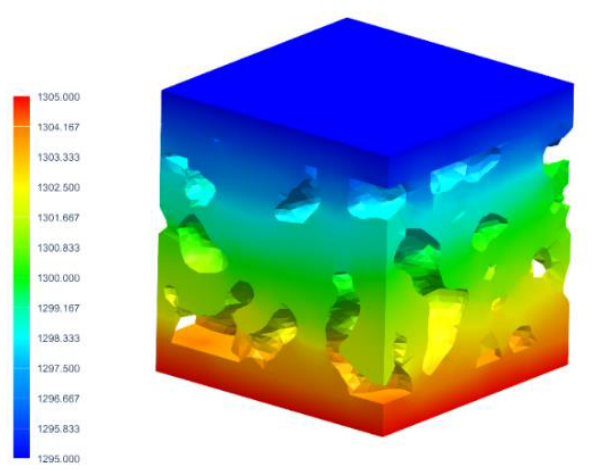

Fig. 7. The thermal state of the representative volume element of the porous CCCM, ${ }^{\circ} \mathrm{C}$.

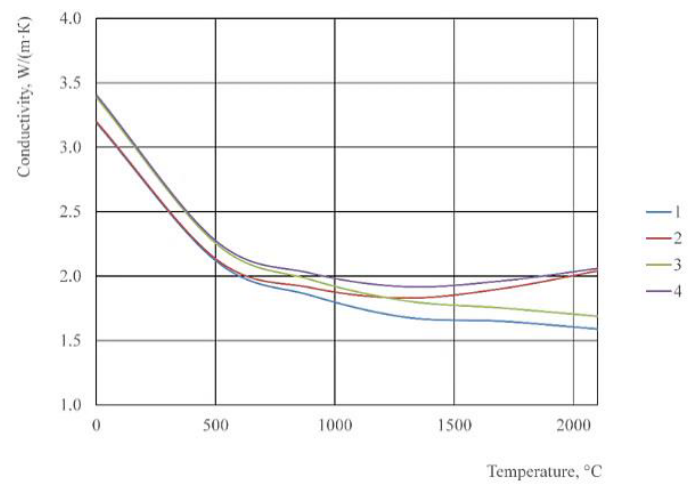

Fig. 8. Molecular $(1,3)$ and effective $(2,4)$ thermal conductivity of the porous CCCM, obtained with the model from the micro-CT scanning $(1,2)$ and a simplified model $(3,4)$.

\section{Estimating the thermal conductivity of the porous CCCM with the simplified model}

Simulating the radiative-conductive heat exchange using the CT data model involves timeand labour-consuming calculations. This is true for the finite-element model stage, and especially true for the calculation stage due to the high complexity of the representative volume element shape.

All of the above brings up the question of creating a simplified structure model that will adequately reproduce its characterisitics and will significantly reduce the time- and labourintensity of the simulation, which is critical at the initial calculation stages.

The MSC.Digimat software package was used to create a structure model comprising the monolithic material and ellipsoid pores with 2:1 semiaxes ratio. Fig. 9 presents the geometric and finite-element simplified structure models; Fig. 10 presents the thermal state of the representative volume element. 

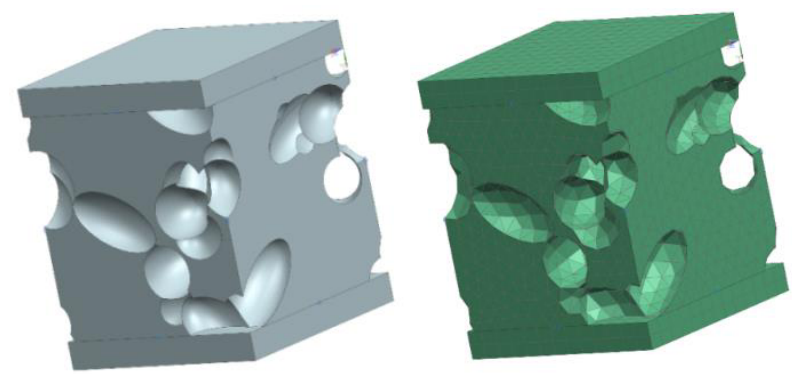

Fig. 9. Finite-element model of the representative volume element of the porous CCCM.

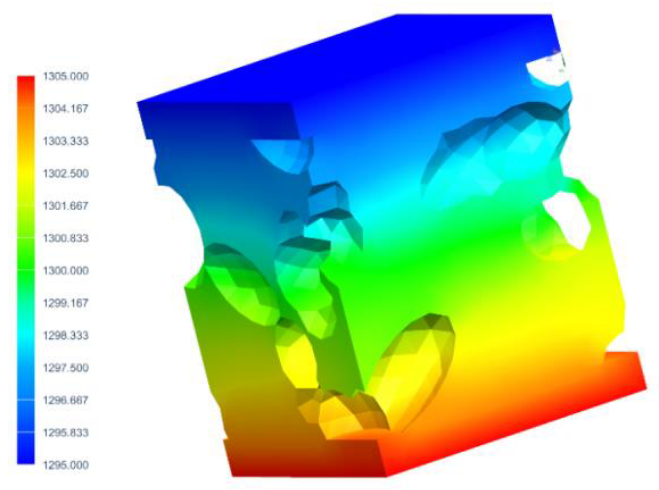

Fig. 10. Thermal state of the representative volume element of the porous $\mathrm{CCCM},{ }^{\circ} \mathrm{C}$.

After that, as in Part 3, the temperature functions of molecular and effective thermal conductivity coefficients were determined for the simplified model (Fig. 8, graphs 3 and 4). It is evident that for the both models, the values of the thermal conductivity coefficient differ by no more than $4 \%$, while the calculation time decreases almost by a factor of 10 . Consequently, the simplified structure model of the porous CCCM can be successfully used for the thermal conductivity calculations of this class materials.

Some results of this paper were obtained as part of the grant agreement No. 14.577.21.0099 by the Russian Federation Ministry of Education and Science. Unique identifier for Applied Scientific Research - RFMEFI57714X0008.

\section{References}

1. P. Narottam, Bansal Handbook of Ceramic Composites (Kluwer academic publishers, Boston, 2005)

2. S. P. Walker, et. al. 20th AIAA International Space Planes and Hypersonic Systems and Technologies Conference (Scotland, UK, 2015)

3. B. Laub, M.J. Wright, E. Venkatapathy, 6th International Planetary Probe Workshop, Atlanta, Georgia Short Course on Extreme Environments Technologies 06/21-22 (2008)

4. S.V. Reznik, P.V. Prosuntsov, K.V. Mikhailovskii, J. Eng. Phys. Thermophys. 88, 3 (2015)

5. P.V. Prosuntsov, N.Y. Taraskin, Matec Web of Conf. 72, 010929 (2016) 